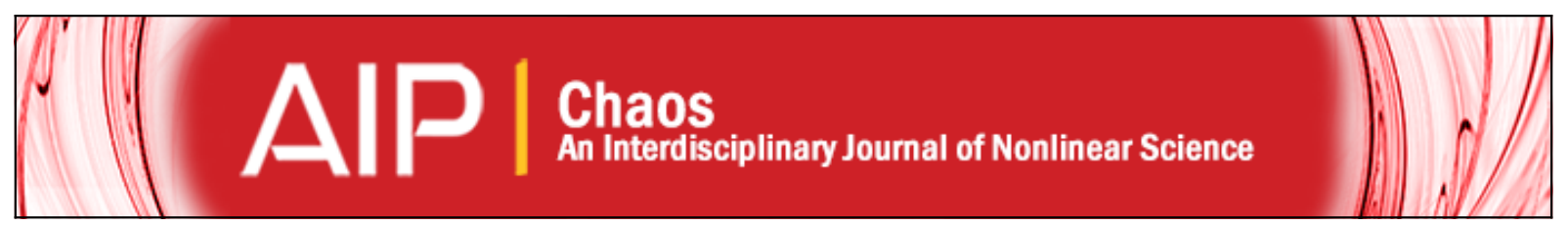

\title{
Control and prediction for blackouts caused by frequency collapse in smart grids
}

Chengwei Wang, Celso Grebogi, and Murilo S. Baptista

Citation: Chaos 26, 093119 (2016); doi: 10.1063/1.4963764

View online: http://dx.doi.org/10.1063/1.4963764

View Table of Contents: http://scitation.aip.org/content/aip/journal/chaos/26/9?ver=pdfcov

Published by the AIP Publishing

\section{Articles you may be interested in}

The optimal home energy management strategy in smart grid

J. Renewable Sustainable Energy 8, 045101 (2016); 10.1063/1.4955045

Demand side management of smart grid: Load shifting and incentives

J. Renewable Sustainable Energy 6, 033136 (2014); 10.1063/1.4885106

Design and experimental investigations of a smart battery energy storage system for frequency control in microgrids

J. Renewable Sustainable Energy 6, 023130 (2014); 10.1063/1.4873995

Effect of plug-in hybrid electric vehicles charging/discharging management on planning of smart microgrid

J. Renewable Sustainable Energy 4, 063144 (2012); 10.1063/1.4772634

Mach-Lorentz Thruster Spacecraft Applications

AIP Conf. Proc. 880, 1063 (2007); 10.1063/1.2437551

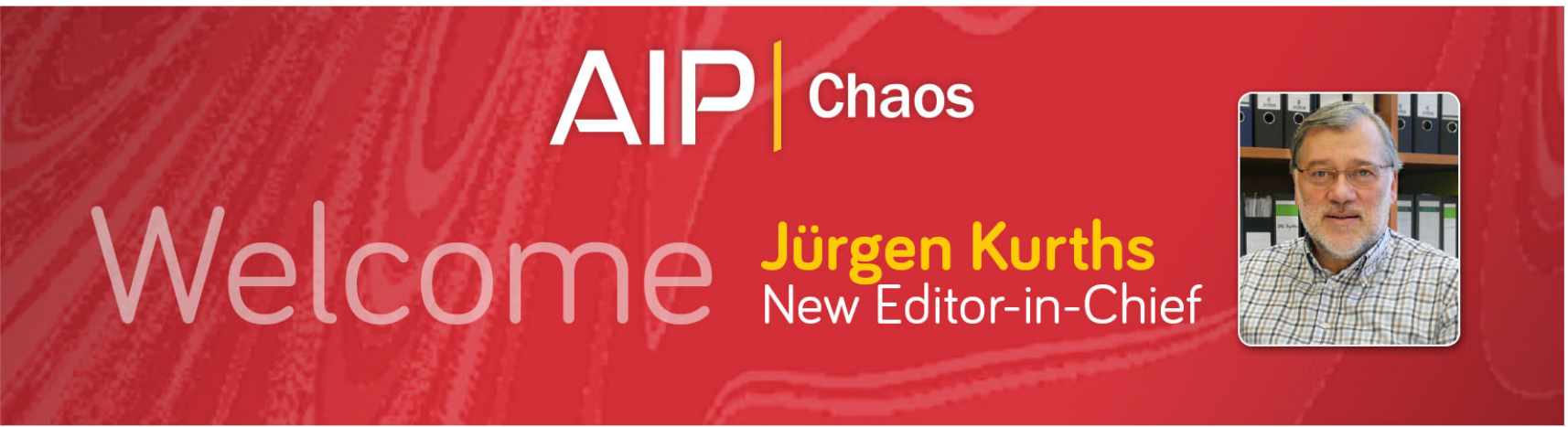




\title{
Control and prediction for blackouts caused by frequency collapse in smart grids
}

\author{
Chengwei Wang, ${ }^{\text {a) }}$ Celso Grebogi, and Murilo S. Baptista \\ Institute for Complex Systems and Mathematical Biology, University of Aberdeen, King's College, \\ AB24 3UE Aberdeen, United Kingdom
}

(Received 3 March 2016; accepted 6 September 2016; published online 30 September 2016)

\begin{abstract}
The electric power system is one of the cornerstones of modern society. One of its most serious malfunctions is the blackout, a catastrophic event that may disrupt a substantial portion of the system, playing havoc to human life and causing great economic losses. Thus, understanding the mechanisms leading to blackouts and creating a reliable and resilient power grid has been a major issue, attracting the attention of scientists, engineers, and stakeholders. In this paper, we study the blackout problem in power grids by considering a practical phase-oscillator model. This model allows one to simultaneously consider different types of power sources (e.g., traditional AC power plants and renewable power sources connected by DC/AC inverters) and different types of loads (e.g., consumers connected to distribution networks and consumers directly connected to power plants). We propose two new control strategies based on our model, one for traditional power grids and another one for smart grids. The control strategies show the efficient function of the fastresponse energy storage systems in preventing and predicting blackouts in smart grids. This work provides innovative ideas which help us to build up a robuster and more economic smart power system. Published by AIP Publishing. [http://dx.doi.org/10.1063/1.4963764]
\end{abstract}

One of the most serious malfunctions of today's electric power grid is the blackout. A blackout is a phenomenon of cascading failures in power grids that may disrupt a substantial portion of power grids, causing large economic losses and impacting on human life. Due to the complexity involved in the modelling of the power grid to understand the basic principles leading to blackouts and ways to control it, research on this topic has attracted the attention of not only engineers but also scientists. In this paper, we study the blackout phenomena resulting from the synchronisation collapse in the generators, by considering a practical phase-oscillator model, which allows one to simultaneously incorporate different types of power sources and loads. We propose two smart control strategies (SCS), one for traditional power grids in which the control of a generator is solely based on its local state and another one for smart grids in which a generator is controlled based on the information about the state of other relevant components of the grids. The control strategies aim to show the active influence on the dynamics of smart grids from the fast-response energy storage systems, which provides an innovative approach to mitigate and predict blackouts in smart grids and to build up a robuster and more economic power system.

\section{INTRODUCTION}

The electrical infrastructure plays a vital significant role in the modern society. The blackout, a phenomenon of cascading failures in power grids, is a comprehensive, complicated and fast-evolving process caused by different reasons. ${ }^{1-3}$ For example, the blackout of the U.S.-Canadian

\footnotetext{
${ }^{a)}$ Electronic mail: r01cw13@abdn.ac.uk
}

power grid on 14 August 2003, interrupted approximately $63 \mathrm{GW}$ of load and affected about 50 million people in eight U.S. states and two Canadian provinces. ${ }^{3,4}$ A nationwide blackout happened in Italy on 28 September 2003, due to cascading failures caused by the tripping of the power transmission line between Italy and Switzerland. ${ }^{5,6}$ On 31 July 2012, a more severe power blackout caused by a relay problem affected 22 states of India and left approximately 700 million people in darkness. ${ }^{7,8}$

A great deal of attention from both the engineers ${ }^{9-14}$ and physicists ${ }^{15-20}$ has recently been drawn to study the blackouts by considering both traditional and smart grids, aiming at finding the most unifying and fundamental reasons for such events. Some works ${ }^{21-23}$ proposed advanced control strategies to prevent these events. Yet, despite these efforts, blackouts are still occurring since power grids are complicated self-organised critical systems $^{24-26}$ experiencing inevitable and diverse levels of disturbances. The adverse influence of a blackout tends to increase, since the modern power grids are expanding with more interconnections among different areas and countries, ${ }^{27}$ making the research for blackout more necessary.

The collapse of frequency synchronisation (FS) in power systems is one of the main causes behind these catastrophic events. Collapsing FS is mainly caused by the imbalance of active power between generators and loads. ${ }^{28,29}$ In a normal operating state, the active power generation and consumption must be timely equal regardless of the power loss in the system. Otherwise, some components of the power grid are tripped due to overload resulting in a disconnection between these components and the main network. A loss of components, such as generators, aggravates the imbalance of active power, which may cause a FS collapse.

In this paper, we discuss the blackout scenarios resulting from an FS collapse, by considering a practical model. 
Comparing to the Kuramoto-like model ${ }^{30}$ and the swing equation, ${ }^{31}$ our model allows one to study power grids by simultaneously considering different types of power sources and different types of consumers. We put forward two smart control strategies to avoid it. One smart control strategy is designed for the traditional power grids in which a generator is controlled based on its own state and another one is for smart grids based on a communication network, which is able to timely collect and exchange information about the state of the network among some important components of power grids. For the latter control strategy, the behaviour of the controlled power system allows us to predict the power energy that the remaining generators need, to prevent a blackout from happening due to a major failure caused by one generator. Our control strategies are based on distributed fast-response energy storage systems, which grant a positive motivation for the development of distributed renewable energy. Comparing to other works, ${ }^{21-23}$ our control strategies can not only prevent a blackout from happening but also greatly decrease the requirement of backup power from generators to restore normal functioning of the power systems. Thus, this work also contributes towards the design and implementation of more resilient and economic power grids.

\section{THE MODEL}

We consider a power grid without power loss in the transmission lines. A reduced power grid can be obtained by the Kron reduction ${ }^{32-34}$ that eliminates all of the junction nodes where the input power is equal to the output power [node 4 in Fig. 1(a)]. Figure 1(b) shows the reduced power grid obtained from the one shown in Fig. 1(a), using the Kron reduction method to eliminate node 4 . In the reduced

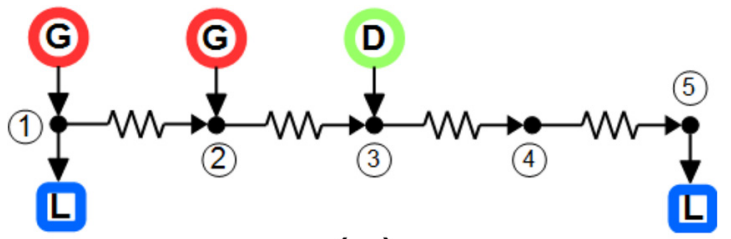

(a)

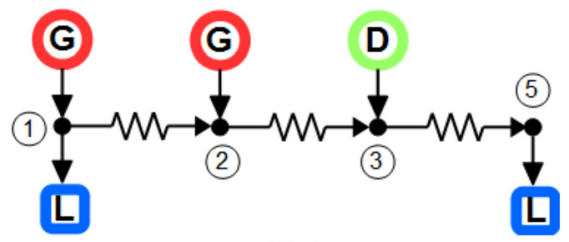

(b)

FIG. 1. (a) The original power grid including 2 generators $(G), 1$ DC source (D), 2 loads (L), and 1 junction node (node 4). (b) The reduced power grid obtained using the Kron reduction to eliminate the junction node.

network, a load may share a node with a generator [node 1 in Fig. 1(b)], or may occupy a separate node [node 5 in Fig. 1(b)]. We use the elements of the index set $\mathcal{I}_{G L}$ $=\left\{1, \ldots, N_{G L}\right\}$ to represent the labels for the nodes indicating generators [node 2 in Fig. 1(b)] or the nodes shared by a generator and a load [node 1 in Fig. 1(b)], the elements of the index set $\mathcal{I}_{D L}=\left\{N_{G L}+1, \ldots, N\right\}$ to denote the labels for the nodes indicating DC sources (e.g., solar power) connected by the DC/AC inverters [node 3 in Fig. 1(b)], or the nodes indicating loads occupying separate nodes [node 5 in Fig. 1(b)], and the elements of the index set $\mathcal{I}_{N}=\{1, \ldots, N\}$ to indicate the labels for all nodes in a reduced power grid.

The model of the power grid is given by Eqs. (1) and (2). The generator is modelled by the swing equation, ${ }^{31}$ namely,

$$
\left\{\begin{array}{l}
\dot{\Theta}_{i}=\Omega_{i}, \quad \forall i \in \mathcal{I}_{G L}, \\
M_{i} \dot{\Omega}_{i}=P_{G, i}+P_{L, i}-\sum_{j=1}^{N} b_{i j} \sin \Theta_{i j}-D_{i} \Omega_{i}, \forall i \in \mathcal{I}_{G L}, \quad \forall j \in \mathcal{I}_{N},
\end{array}\right.
$$

where $\Theta_{i}$ is the phase angle of node $i, \Theta_{i j}=\Theta_{i}-\Theta_{j}, \Omega_{i}$ is the instantaneous angular frequency of generator $i, M_{i}$ and $D_{i}$ are the normalised inertia and damping coefficient, respectively, $P_{G, i}$ is the mechanical power provided by turbine $i, P_{L, i}$ is the power consumed by the load sharing node $i$, and $P_{L, i}=0$ if there is no load sharing the node with generator $i ; b_{i j}=\left|U_{i}\right|\left|U_{j}\right| \Im Y_{i j}$, where $U_{i}$ is the bus voltage of node $i, Y_{i j}=Y_{j i}$ is a complex number representing the admittance of the transmission line between nodes $i$ and $j$; $Y_{i j}=0$ if $i$ and $j$ are not directly connected in the reduced power grid and $\Im Y_{i j}$ is the imaginary part of $Y_{i j}$.

A DC source or a load occupying a separate node is modelled by ${ }^{14,35}$

$$
D_{i} \dot{\Theta}_{i}=P_{D L, i}-\sum_{j=1}^{N} b_{i j} \sin \Theta_{i j}, \quad \forall i \in \mathcal{I}_{D L}, \quad \forall j \in \mathcal{I}_{N}
$$

where for a DC source, $P_{D L, i}>0$ is the nominal power source, $1 / D_{i}$ is the droop-slope of the droop controller of the DC/AC inverter; for a load occupying a separate node, $P_{D L, i}<0$ is a constant power load, $D_{i}>0$ and $D_{i} \dot{\Theta}_{i}$ is a part of frequencydependent load.

We apply a rotating frame for the models in Eqs. (1) and (2) by letting $\theta_{i}=\Theta_{i}-\Omega_{n} t$ and $\omega_{i}=\Omega_{i}-\Omega_{n}$, where $\Omega_{n}=2 \pi f_{n}$ is the natural angular frequency, and $f_{n}=50 \mathrm{~Hz}$ or $60 \mathrm{~Hz}$ is the natural frequency of the power grid. The natural angular frequency becomes $\omega_{n}=0$ in the rotating frame, then Eqs. (1) and (2) become 


$$
\left\{\begin{array}{l}
\dot{\theta}_{i}=T_{D L, i}-\sum_{j=1}^{N} a_{i j} \sin \theta_{i j}, \forall i \in \mathcal{I}_{D L}, \forall j \in \mathcal{I}_{N}, \\
\dot{\theta}_{i}=\omega_{i}, \forall i \in \mathcal{I}_{G L}, \\
\dot{\omega}_{i}=T_{G, i}+T_{L, i}-\sum_{j=1}^{N} a_{i j} \sin \theta_{i j}-F_{i} \omega_{i}, \forall i \in \mathcal{I}_{G L}, \forall j \in \mathcal{I}_{N},
\end{array}\right.
$$

where $\theta_{i j}=\theta_{i}-\theta_{j}$; if $i \in \mathcal{I}_{D L}, T_{D L, i}=\frac{P_{D L, i}}{D_{i}}-\Omega_{n}, a_{i j}=\frac{b_{i j}}{D_{i}}$; if $i \in \mathcal{I}_{G L}, T_{G, i}=\frac{P_{G, i}}{M_{i}}, T_{L, i}=\frac{1}{M_{i}}\left(P_{L, i}-D_{i} \Omega_{n}\right), a_{i j}=\frac{b_{i j}}{M_{i}}, F_{i}=\frac{D_{i}}{M_{i}}$.

Equation (3) describes a power grid of a coupled phaseoscillator network which contains both traditional AC power plants and renewable power sources connected by DC/AC converters, and includes both users connected to distribution networks and consumers powered directly by power plants. This model actually can be treated as a transformation of the structure-preserving model (the SP model) proposed in Ref. 36. A steady state of the power grid corresponds to a FS state of Eq. (3), defined by $\dot{\theta}_{i}=\omega_{i}=0$. Summing the first and third equations, which are related to power transmission in Eq. (3) for all $i$, we have

$$
\begin{aligned}
\sum_{i \in \mathcal{I}_{G L}} \dot{\omega}_{i}+\sum_{i \in \mathcal{I}_{D L}} \dot{\theta}_{i}= & \sum_{i \in \mathcal{I}_{G L}} T_{G, i}+\sum_{i \in \mathcal{I}_{G L}} T_{L, i} \\
& +\sum_{i \in \mathcal{I}_{D L}} T_{D L, i}-\sum_{i \in \mathcal{I}_{G L}} F_{i} \omega_{i}
\end{aligned}
$$

In a steady state, the power system operates at an equilibrium point, and all nodes are in frequency synchronisation with $\dot{\theta}_{i}^{e q}=\omega_{i}^{e q}=\omega_{n}=0$, implying the "imbalance power" between generators and loads to be zero, namely,

$$
d T=\sum_{i \in \mathcal{I}_{G L}} T_{G, i}+\sum_{i \in \mathcal{I}_{G L}} T_{L, i}+\sum_{i \in \mathcal{I}_{D L}} T_{D L, i}=0 .
$$

This means that the power produced by generators is equal to that consumed by loads in a steady state.

Blackout process: Assume that at $t=t_{0}$ there is a loss of a high-capacity generator with label $m \in \mathcal{I}_{G L}$, i.e., $T_{G, m}$ suddenly becomes 0 from a large positive value, such that $d T$ $<0$. The stored kinetic energy in the rotors of all remaining generators are then released to balance the power between generators and consumers, resulting in the deceleration of the speed of rotors, i.e., a drop of the angular frequencies $\omega_{i}$ from 0 . In order to maintain the stability of the system, the remaining generators need to provide additional power, such that $d T$ returns to 0 and all $\omega_{i}$ also returns to 0 . The power system then reaches a new steady state. The regulation process of the output power in a generator can be controlled by its active power regulating system, which is described by

$$
\dot{T}_{G, i}=-K_{i} \omega_{i}, \quad \forall i \in \mathcal{I}_{G L},
$$

where $K_{i}>0$ is the regulation constant of generator $i$ that can be manually set, and $\omega_{i}$ indicates the frequency deviation from 0 for node $i$ in the dynamic process.

Equation (6) can represent either the turbine governor system or the energy storage system in power grids. The mechanism of this control is that when $d T<0(>0)$, the angular speed of the generator rotors, $\omega_{i}$ decreases (increases) due to the release (accumulation) of kinetic energy in the rotors. This leads to a negative (positive) $\omega_{i}$, which, according to Eq. (6), forces $T_{G, i}$ to increase (decrease) by automatically turning up (down) the flow rate of steam into the turbine. Thus, more (less) energy is provided by prime movers. The adjusting power from prime movers accelerates (decelerates) the rotors to balance the generation power and consumption power. Finally, $\omega_{i}$ returns to $0, d T$ becomes 0 as well, and the whole system reaches a new steady state. By providing such a negative feedback to the system, this control enhances the stability of the system around its equilibrium point. Traditionally, the turbine governor system needs long time to adjust the flow rate of steam due to the mechanical inertia of machines. However, some new energy storage systems are developed nowadays, ${ }^{37,38}$ such as large battery arrays, solar farms, and the storage systems in wind farms, to provide faster response to the frequency change and quickly provide supplementary power into the system to help it to reach a new steady state. In this paper, we assume all the energy storage systems in power plants are fast-response systems.

When the system loses generator $m$, the angular frequencies of the remaining generators fluctuate, and these generators provide supplementary power according to Eq. (6) in order to mitigate the frequency fluctuation. Let $T_{G, i}^{\max }$ and $T_{G, i}^{\min }$ be the upper and lower physical bounds of $T_{G, i}$. During the dynamic process, if $T_{G, i}>T_{G, i}^{\max }$ (overload) or $T_{G, i}<T_{G, i}^{\min }$, generator $i$ is tripped by its protection devices, resulting in a disconnection of an additional generator. A loss of one more generator results in larger disturbance of the whole system and more power requirement from other generators. This leads more generators to be tripped due to overload. Such a cascading failure may lead to a FS collapse, resulting in a blackout in the power grid, i.e., the loss of all generators. The backup-power capacity of generator $i$ is defined by

$$
T_{b, i}=T_{G, i}^{\max }-T_{G, i}^{e q} .
$$

where $T_{G, i}^{e q}$ is the value of $T_{G, i}$ when the system gets into a steady state, namely, a frequency synchronisation state among all phase-oscillators described in Eq. (3). We define the power ratio, $\eta_{i}$, to indicate the ratio between the power supplemented by generator $i$ and its output power in the steady state before $t_{0}$ by

$$
\eta_{i}=\frac{T_{G, i}-T_{G, i}^{e q}}{T_{G, i}^{e q}}
$$


(a)
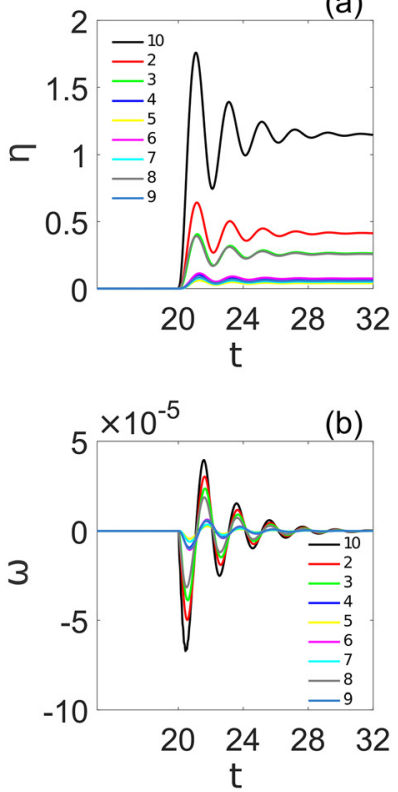

(c)
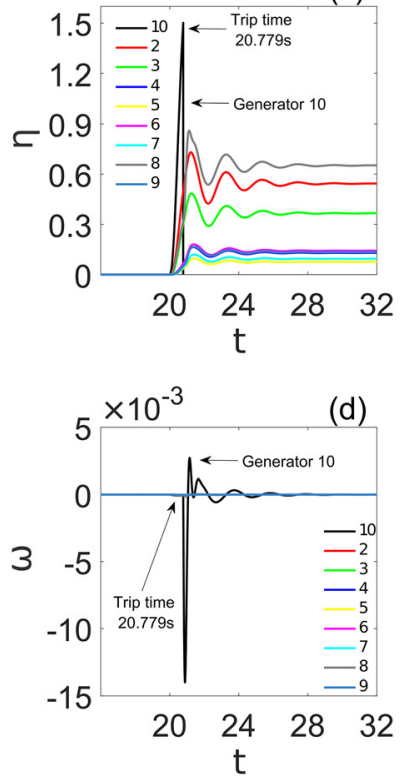

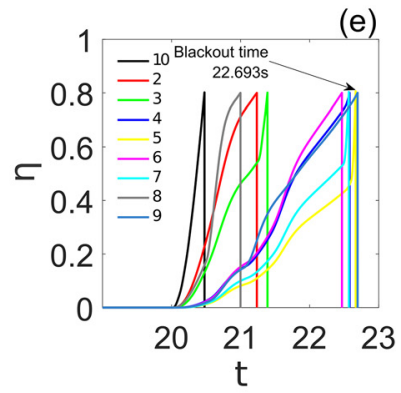

(f)

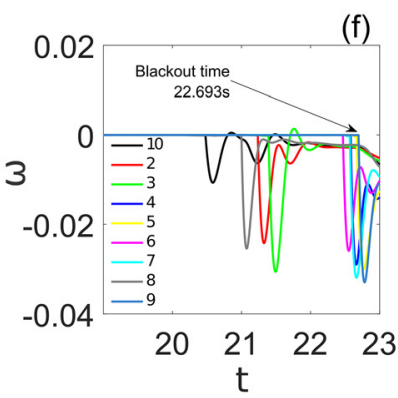

FIG. 2. Simulation for the IEEE 39 bus system. (a) and (b) The changes of the power ratio, $\eta_{i}$, and the angular frequency, $\omega_{i}, \quad$ respectively, with $\eta_{i}^{\max }=2$; (c) and (d) the changes of $\eta_{i}$ and $\omega_{i}$, respectively, with $\eta_{i}^{\max }=1.5$; (e) and (f) the changes of $\eta_{i}$ and $\omega_{i}$, respectively, with $\eta_{i}^{\max }=0.8$.

Let

$$
\eta_{i}^{\max }=\frac{T_{G, i}^{\max }-T_{G, i}^{e q}}{T_{G, i}^{e q}}=\frac{T_{b, i}}{T_{G, i}^{e q}} \geq 0
$$

and

$$
\eta_{i}^{\min }=\frac{T_{G, i}^{\min }-T_{G, i}^{e q}}{T_{G, i}^{e q}} \leq 0
$$

represent the maximum and minimum values that $\eta_{i}$ can assume, respectively. We simply set $\eta_{i}^{\min }=-0.5$ (i.e., $\left.T_{G, i}^{\min }=0.5 T_{G, i}^{e q}\right), \forall i \in \mathcal{I}_{G L}$, because we focus on the overload problem in this paper and $\eta_{i}^{\min }$ does not affect our numerical experiments.

We use the IEEE 39 bus system to show how blackout happens and how $\eta_{i}^{\max }$ affects the behaviour of the system. The IEEE 39 bus system, also known as the New-England Power System, includes 10 generators, 2 loads sharing nodes with generators, 17 loads occupying separate nodes, and 12 junction nodes. Appendix provides the topology and the data required in numerical experiments for this system. By Kron reduction, ${ }^{32-34}$ we eliminate the junction nodes and obtain a system with 27 nodes. In the simulation, we only consider the frequency collapse as the reason of a blackout, by manually setting an ideal environment which is obtained by neglecting the effects of other reasons. For instance, we assume that all power lines are strong enough and have sufficient capacities to transfer the power flowing on them, and that there is enough reactive power provided by local reactive power suppliers to maintain the voltage level, ${ }^{39}$ such that all coupling strengths $\left(a_{i j}\right)$ remain unchanged in the simulation. This experimental environment is not $100 \%$ as real as that in the practical power grids, but it allows us to better understand the mechanism of the collapse of frequency synchronisation as a main reason of blackouts, and to develop the smart control strategies. We initiate the dynamic process by switching off generator 1 which has the maximum

capacity at $\mathrm{t}=20 \mathrm{~s}$, i.e., forcing $T_{G, 1}=0$ at $t=20 \mathrm{~s}$. We plot the changes of $\eta_{i}$ and $\omega_{i}$ for the remaining generators. We set $T_{G, i}=0$ and $\eta_{i}=0$ if generator $i$ is tripped due to overload in the experiments. Figures 2(a) and 2(b) show the results with a large $\eta_{i}^{\max }=2.0, \forall i \in \mathcal{I}_{G L}$. Every generator supplements some power from $t=20 \mathrm{~s}$ and no remaining ones are tripped [Fig. 2(a)]. The angular frequency of each generator, $\omega_{i}$, experiences fluctuation from $t=20 \mathrm{~s}$, but finally returns to 0 [Fig. 2(b)], meaning that the system reaches a new steady state. Figures 2(c) and 2(d) show simulations considering $\eta_{i}^{\max }=1.5, \forall i \in \mathcal{I}_{G L}$. Figure 2(c) indicates that generator 10 is tripped at $t=20.779 \mathrm{~s}$ due to overload, but other generators successfully provide enough power to the system. Thus, the system reaches a new steady state, i.e., the angular frequencies of the remaining generators finally become 0 , as shown in Fig. 2(b). Figures 2(e) and 2(f) show the results with $\eta_{i}^{\max }=0.8, \forall i \in \mathcal{I}_{G L}$. As shown in Fig. 2(e), the generators are tripped one by one due to overload. Finally, at $t=22.693 \mathrm{~s}$, the system lose all generators and a FS collapse occurs [Fig. 2(f)].

\section{SMART CONTROL}

\section{A. Smart control I}

As shown in Figs. 2(c) and 2(e), when a generator is tripped due to overload, the $\eta_{i}$ of the remaining generators are still far away from the maximum limit, meaning that the remaining generators still possesses large amounts of backup power that can be used to restore the stability of the power grid. In order to efficiently use the backup power of every generator to avoid a blackout, we develop a smart control, which will greatly improve the robustness of power grids with less requirement of backup power for generators. For that, we change Eq. (6) into

$$
\dot{T}_{G, i}=-\alpha_{i} K_{i} \omega_{i}, \quad \forall i \in \mathcal{I}_{G L}
$$


(a)
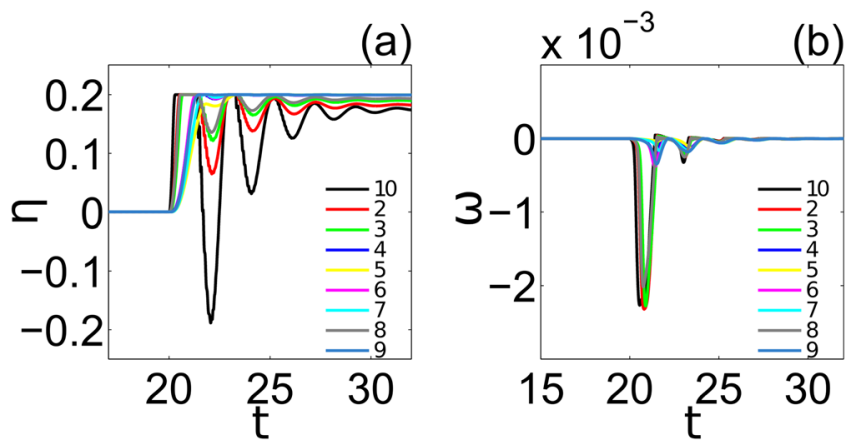

FIG. 3. Results for the IEEE 39 bus system with smart control I. (a) and (b) The changes of the power ratio, $\eta_{i}$, and the angular frequency, $\omega_{i}$, respectively, with $\eta_{i}^{\max }=0.2$.

where $\alpha_{i}=\left(T_{G, i}^{\max }-T_{G, i}\right) /\left(T_{G, i}^{\max }-T_{G, i}^{e q}\right)$ if $\omega_{i} \leq 0$, and $\alpha_{i}$ $=\left(T_{G, i}-T_{G, i}^{\min }\right) /\left(T_{G, i}^{e q}-T_{G, i}^{\min }\right)$ if $\omega_{i}>0$. At a steady state, $T_{G, i}=T_{G, i}^{e q}$, we have $\alpha=1$; when generator $i$ reaches its output limits, we have $T_{G, i}=T_{G, i}^{\max }$ or $T_{G, i}=T_{G, i}^{\min }$ resulting in $\alpha_{i}=0$. Thus, $T_{G, i}$ does not change any more when it reaches its limits, and none of the generators are then tripped due to overload.

The utilisation ratio of the backup-power capacity of generator $i$ is defined by

$$
\sigma_{i} \%=\frac{T_{G, i}-T_{G, i}^{e q}}{T_{b, i}} .
$$

Figure 3 and Table I show the numerical results for the IEEE 39 bus system with smart control I. Set $\eta_{i}^{\max }=0.2, \forall i \in \mathcal{I}_{G L}$, which is smaller than $\eta_{i}^{\max }=0.8, \forall i \in \mathcal{I}_{G L}$ that was used in Figs. 2(e) and 2(f) where a blackout happens without the implementation of smart control I. A smaller $\eta_{i}^{\max }$ indicates a smaller backup-power capacity of generator $i$. At $t=20 \mathrm{~s}$, we lose generator 1. With our control strategy, none of the remaining generators is tripped, although some of them have almost provided their full backup-power capacity (some $\sigma_{i} \% \approx 100 \%$ in Table I), and the angular frequencies of all remaining generators return to 0 after some fluctuations [Fig. 3(b)]. This means that, by applying the smart control I, we avoid a blackout in the system with less backup-power capacity requirement for generators. Less backup-power capacity requirement greatly improves the economic side of power systems.

\section{B. Smart control II}

The smart control I is easily implemented because $\omega_{i}$ and $\alpha_{i}$ can be locally measured or calculated for every generator. However, the drawback of the smart control I is that some of the generators nearly reach their maximum output limits (some $\sigma_{i} \% \approx 100 \%$ in Table I), reserving no extra power for engineers to impose any further manual control with these generators and leaving these generators at a dangerous critical

TABLE I. Utilisation ratio of backup power of generators with smart control I.

\begin{tabular}{cccccccccc}
\hline \hline$i$ & 10 & 2 & 3 & 4 & 5 & 6 & 7 & 8 & 9 \\
\hline$\sigma_{i} \%$ & 87.9 & 91.4 & 94.9 & 99.7 & 99.8 & 99.6 & 99.8 & 96.7 & 99.7 \\
\hline \hline
\end{tabular}

(a)

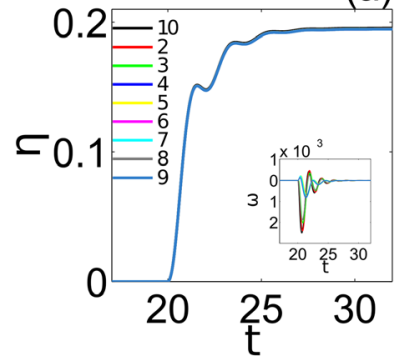

(b)

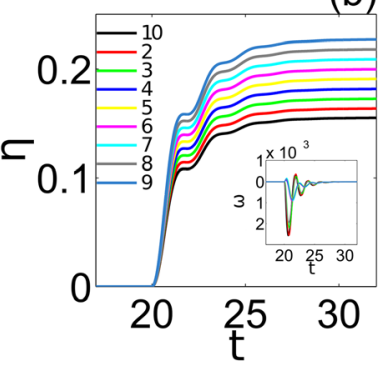

FIG. 4. Simulation for the IEEE 39 bus system with the smart control II. (a) The change of the power ratio, $\eta_{i}$, by the main plot and the angular frequency, $\omega_{i}$, by the sub-plot with $\eta_{i}^{\max }=0.2, \forall i \in \mathcal{I}_{G L}$; (b) the changes of $\eta_{i}$ (main plot) and $\omega_{i}$ (sub-plot) with $\eta_{i}^{\max }$ varying from 0.17 to 0.25 .

state. Next, we propose an improved smart control based on the smart grid technology to tackle this problem. With the fast development of smart grids, it becomes possible to timely measure and exchange the information (e.g., $\omega_{i}$ ) among different nodes in a power network by a separate network layerthe communication network. ${ }^{14,40}$ To utilise the communication network, we change Eq. (6) into

$$
\dot{T}_{G, i}=-\beta_{i} K_{i} \bar{\omega},
$$

where $\beta_{i}=T_{b, i} / T_{b}^{\max }$ with $T_{b}^{\max }:=\max \left\{T_{b, i} \mid i \in \mathcal{I}_{G L}\right\}$ indicating the maximum backup-power capacity among all generators, and $\bar{\omega}=\sum_{i=1}^{N} \gamma_{i} \omega_{i} / \sum_{i=1}^{N} \gamma_{i}$ with $\gamma_{i}$ indicating the importance level of node $i$. We set $\gamma_{i}=1$ if node $i$ is a generator, a large capacity DC source, or an important load which is sensitive to frequency change, and $\gamma_{i}=0$ if the information of node $i$ is unavailable or node $i$ is not important (e.g., a normal load).

Smart control II improves the control performance by introducing the average angular frequency, $\bar{\omega}$, which embodies a teamwork principle, i.e., one generator lost, all the remaining generators supplement required power together, according to the change in the average angular frequency of some important nodes instead of according to their local angular frequencies. Furthermore, the new variable, $\beta_{i}$, prompts generator $i$ to provide power based on its backuppower capacity, $T_{b, i}$. In other words, a generator with a larger backup-power capacity contributes more power to the power grid than the one with a smaller capacity. This is also an improvement compared to the smart control $\mathrm{I}$ in which the variable $\alpha_{i}$ just limits the maximum output of generator $i$ to ensure its non-overload.

Figure 4 and Table II demonstrate the effectiveness of smart control II. At $t=20 \mathrm{~s}$, we lose generator 1. Figure 4(a) shows the results with $\eta_{i}^{\max }=0.2, \forall i \in \mathcal{I}_{G L}$. The nine lines

TABLE II. Utilisation ratio of backup power of generators with smart control II.

\begin{tabular}{lccccccccc}
\hline \hline$i$ & 10 & 2 & 3 & 4 & 5 & 6 & 7 & 8 & 9 \\
\hline$\eta_{i}^{\max }$ & & & & & 0.20 & & & & \\
$\sigma_{i} \%$ & 97.7 & 97.4 & 97.3 & 97.3 & 97.4 & 97.3 & 97.4 & 97.4 & 97.3 \\
$\eta_{i}^{\max }$ & 0.17 & 0.18 & 0.19 & 0.20 & 0.21 & 0.22 & 0.23 & 0.24 & 0.25 \\
$\sigma_{i} \%$ & 91.2 & 90.9 & 90.8 & 90.8 & 90.9 & 90.8 & 90.9 & 90.9 & 90.8 \\
\hline \hline
\end{tabular}


indicating $\eta_{i}$ for the nine remaining generators in Fig. 4(a) merges into one and $\sigma_{i} \% \approx 97 \%$ for $i=2, \ldots, 10$, as shown in Table II, meaning that all generators supplement power with the same ratio to their back-up power capacities. Finally, none of the remaining generators reaches their full output limits, and the frequencies finally return to 0 as shown in the subplot in Fig. 4(a). In real power systems, $\eta_{i}^{\max }$ is not strictly equal to $\eta_{j}^{\max }$ for $i \neq j$. Figure 4(b) shows the result for a more realistic case where $\eta_{i}^{\max }$ varies from 0.17 to 0.25 (shown in Table II). In this case, $\sigma_{i} \% \approx 91 \%$ for $i=2, \ldots, 10$ (shown in Table II), meaning that all remaining generators still provide power with the same proportion to their own backup-power capacities, even though $\eta_{i}^{\max }$ is different. Our numerical experiments indicate that smart control II not only avoids a blackout but also prevents some generators from reaching their critical points, which greatly improves the stability and robustness of the IEEE 39 bus system.

By comparing Figs. 2(a) and 4, we conclude that smart control II (Fig. 4) restrains oscillations on the curves of $\eta$. This means that, with the implementation of the smart control II, the remaining generators do not need to provide more power in the dynamic process than that required in the final steady state of the power system. Thus, the back-up power capacity of generators can be decreased by implementing smart control II.

In conclusion, smart control II improves the economic side of a power system, since in total less power is required to recover the power system from a dynamic state to a steady state. In other words, less back-up power capacity is required in the whole system in order to maintain its stable functioning. This helps engineers to reduce their budget when they design, build up, and extend a power grid by choosing the energy storage with a relatively smaller capacity.

\section{PREDICTING BLACKOUTS}

Assume that the generator $m$ with capacity $T_{m}$ is lost. Smart controls I and II enable the remaining generators to provide their full back-up power capacities. As a consequence, we can predict that a blackout happens if the total back-up power $\sum_{i \neq m} T_{b, i}$ cannot match the lost capacity, i.e., if $\sum_{i \neq m} T_{b, i}$ $<T_{m}$. Furthermore, smart control II allows the remaining generators to provide power with nearly the same ratio $(\sigma \%$ $=\sigma_{i} \% \approx \sigma_{j} \%$, for $i \neq j$ ) as their back-up power capacities, $T_{b, i}$. This means that $T_{m}=\sum_{i \neq m} \sigma_{i} \% T_{b, i} \approx \sigma \% \sum_{i \neq m} T_{b, i}$, i.e., the utilisation ratio of the back-up power capacity for every remaining generator can be approximately obtained by

$$
\sigma \% \approx \frac{T_{m}}{\sum_{i \neq m} T_{b, i}} .
$$

Thus, we can predict, without numerical simulation, how much power is finally provided by each remaining generator by changing Eq. (12) to

$$
T_{G, i}=\sigma \% T_{b, i}+T_{G, i}^{e q}
$$

where $T_{b, i}$ can be calculated from Eq. (9) with known $\eta_{i}^{\max }$ and $T_{G, i}^{e q}$.
TABLE III. The relative errors between the predicted and numerical values of $T_{G, i}$ with smart control II.

\begin{tabular}{lccccccccc}
\hline \hline$i$ & 10 & 2 & 3 & 4 & 5 & 6 & 7 & 8 & 9 \\
\hline$\eta_{i}^{\max }$ & & & & & 0.20 & & & & \\
$\delta_{i} / 10^{-4}$ & 5.4 & 0.3 & 1.3 & 1.3 & 0.3 & 1.3 & 0.3 & 0.3 & 1.3 \\
$\eta_{i}^{\max }$ & 0.17 & 0.18 & 0.19 & 0.20 & 0.21 & 0.22 & 0.23 & 0.24 & 0.25 \\
$\delta_{i} / 10^{-4}$ & 4.8 & 0.4 & 1.1 & 1.2 & 0.5 & 1.3 & 0.5 & 0.5 & 1.5 \\
\hline \hline
\end{tabular}

Define the relative error between the predicted and the numerical obtained values of $T_{G, i}$ by $\delta_{i}$ $=\left|\left(T_{G, i}^{\prime}-T_{G, i}\right) / T_{G, i}\right|$, where $T_{G, i}^{\prime}$ indicates the predicted value of $T_{G, i}$ from Eq. (15). We carry out two numerical simulations similar to the previous ones. We set $\eta_{i}^{\max }$ $=0.2, \forall i \in \mathcal{I}_{G L}$ for one case, and set $\eta_{i}^{\max }$ varying from 0.17 to 0.25 for another case. At $t=20 \mathrm{~s}$, we lose generator 1. We record the numerical output power of the remaining generators after the system is restored. Table III demonstrates the values of $\delta_{i}$ in the simulations. All the values of $\delta_{i}$ are small, which means that our prediction is effective.

The significance of developing this predicting method is that engineers can now be aware of the weak parts of the system before a cascading failure happens by predicting whether a generator will overload or not during a system fault. Thus, preventive actions, such as adding new energy storages or reallocating the power supplied among different power plants, can be applied with a clear target in order to enhance the stability of the power systems. With the increasing size of the modern power grids, such a targeted preventive action will greatly save the labour and economic cost than healing the power grids from a whole system level.

\section{CONCLUSION}

In this paper, we have studied the mechanism that creates blackouts in a realistic model for the power grid due to a loss of synchronisation among the generators. Based on this study, we provided two smart control strategies which require less backup power for the generators to avoid the onset of a blackout. One of the smart control strategies was used for the traditional power systems, in which the control of a generator is only based on its own state; the other control was designed for the smart grids, in which the control of a generator considers the state of other generators. For the latter control strategy, the behaviour of the controlled power system allowed us to predict the power energy that the remaining generators needed, to prevent a blackout from happening due to a major failure caused by one generator. Our control strategies demonstrate the active influence of the distributed fast-response storage systems in smart grids.

For simplicity, we applied our control methods on the nodes representing generators in the model described by Eq. (3). However, we note that the smart control methods, incorporated in Eqs. (11) and (13), can also be applied to other nodes, such as the nodes representing the DC sources described in Eq. (2), provided that these nodes possess fastresponse back-up power devices. This extension is of great significance in applications, because it means that the 


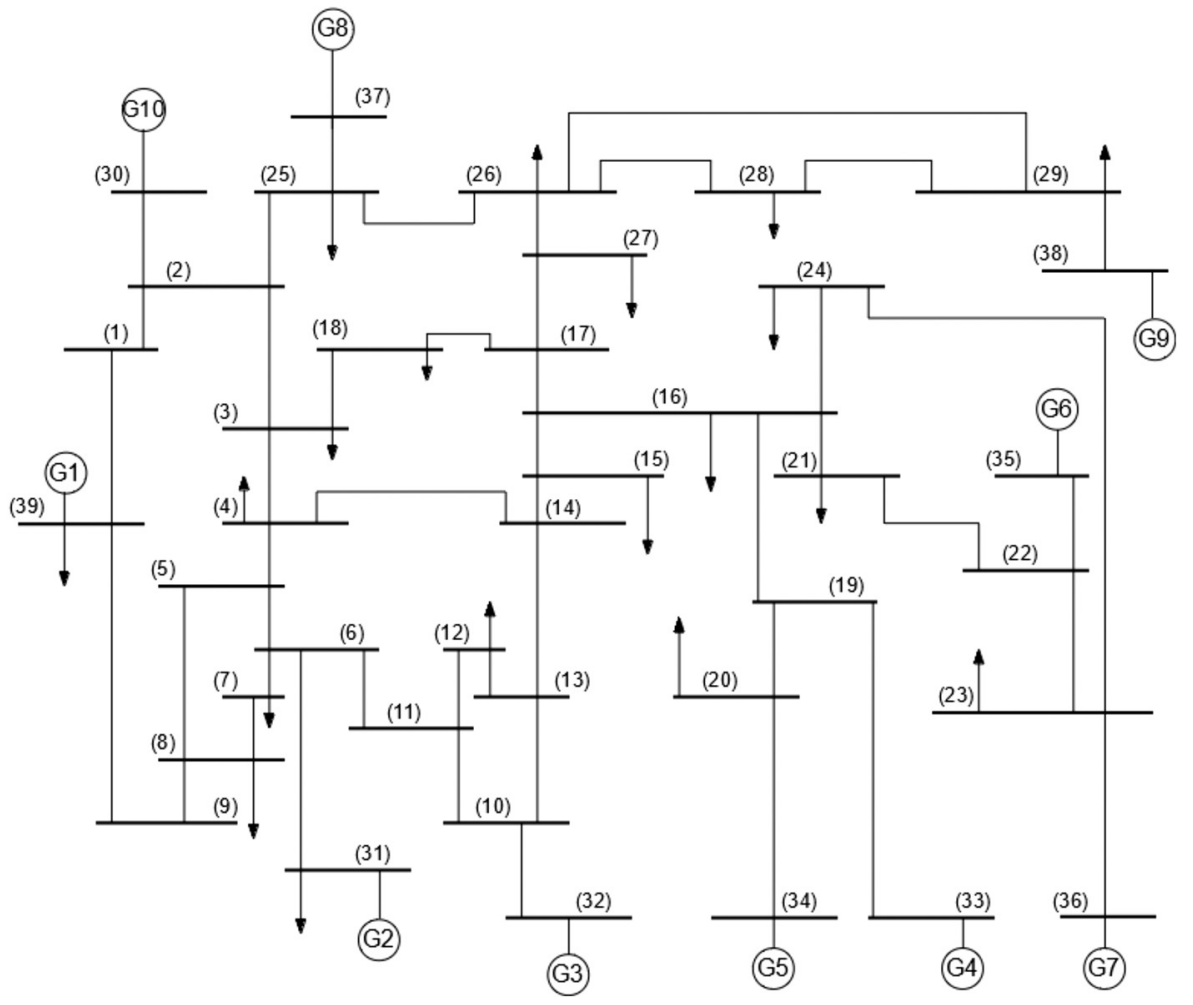

FIG. 5. Diagram of the IEEE 39 bus system, where the numbers in brackets are bus labels and "Gi" in circle indicates generator $i$. renewable energy systems, such as wind farms and solar farms, could also be dealt with the control scheme. Thus, our methods greatly contribute to the design of the control scheme on a system level for the future smart grids where renewable sources are increasingly integrated.

We considered the IEEE 39 bus system as a practical topology for numerical simulations in this work, instead of an abstract topological model of the power grids, such as small world networks or random networks. Noticing that our control methods are applied to the fast-response energy storage systems in the power grids, regardless of the topologies of the networks, we can safely conclude that our control scheme is applicable to the fast-response energy storage devices located anywhere in a connected power grid possessing an arbitrary topology.

In conclusion, our work contributes for the understanding of power grids by studying a more practical model, also helps engineers to improve the robustness and economic aspects of power grids by applying the smart control strategies.

\section{ACKNOWLEDGMENTS}

C.-W.W. was supported by a studentship funded by the College of Physical Sciences, University of Aberdeen. M.S.B. acknowledges EPSRC Grant No. EP/I032606/1.

\section{APPENDIX: THE IEEE 39 BUS SYSTEM}

The topology of the IEEE 39 bus system is shown in Fig. 5. We treat every bus as a node; thus, there are 39 nodes in this system including 10 generators, 17 consumers occupying separated nodes, 2 consumers sharing nodes with generators (buses 31 and 39), and 12 junction nodes. This power grid can be reduced to a 27 node network by eliminating the 12 junction nodes through Kron reduction. In our analysis, we use $N^{0}=39$ and $N=27$ to represent the total number of nodes in the original network and in the reduced network, respectively.

Tables IV and V illustrate the data for buses and transmission lines, respectively. Reference 41 provides a power flow study result for the IEEE 39 bus system, which contains all the original data, except for the damping coefficient $D$ and the control parameter $K$, for our numerical experiments. We set $D_{i}=2, \forall i \in \mathcal{I}_{G L}, D_{i}=3, \forall i \in \mathcal{I}_{D L}$. Let $K_{i}=1$, $\forall i \in \mathcal{I}_{G L}$ for the numerical experiments without smart control strategy (SCS) and with SCS I, and $K_{i}=0.01, \forall i \in \mathcal{I}_{G L}$ for the numerical experiments with SCS II, since generators become more sensitive to the frequency change with SCS II and the numerical experiments cannot get a convergent result with $K=1$. The normalised inertia can be calculated by $M_{i}=\frac{2 H_{i}}{\Omega_{n}}=\frac{2 H_{i}}{2 \pi f_{n}}$, where $f_{n}=50 \mathrm{~Hz}$ or $60 \mathrm{~Hz}$ is the natural frequency of power systems. $P_{G}^{0}$ and $P_{L}^{0}$ are obtained from the power flow results in Ref. 41 by considering the power loss in transmission lines, resulting in $\sum_{i=1}^{N_{G}} P_{G, i}^{0}+\sum_{i=N_{G}+1}^{N}$ $P_{L, i}^{0}=43.71 \neq 0$, where 43.71 indicates the total power loss in the transmission lines. In order to construct a reduced network (27 nodes) without power loss in the transmission line, we let $P_{G, i}=P_{G, i}^{0}-\bar{P}$ and $P_{L, i}=P_{L, i}^{0}-\bar{P}$, where $\bar{P}=\frac{1}{N}$ $\left(\sum_{i=1}^{N_{G}} P_{G, i}^{0}+\sum_{i=N_{G}+1}^{N} P_{L, i}^{0}\right)=\frac{43.17}{27} \approx 1.6189$. This means that the 27 nodes in the reduced network equally share the power loss in the transmission lines, such that $\sum_{i=1}^{N_{G}} P_{G, i}$ $+\sum_{i=N_{G}+1}^{N} P_{L, i}=0$, indicating that the power provided by generators are equal to that consumed by consumers. 
TABLE IV. Transmission line data. Data from Ref. 41

\begin{tabular}{|c|c|c|c|c|}
\hline From bus & To bus & $\mathrm{R}$ & $X$ & B \\
\hline 1 & 2 & 0.0035 & 0.0411 & 0.6987 \\
\hline 1 & 39 & 0.0010 & 0.0250 & 0.7500 \\
\hline 2 & 3 & 0.0013 & 0.0151 & 0.2572 \\
\hline 2 & 25 & 0.0070 & 0.0086 & 0.1460 \\
\hline 3 & 4 & 0.0013 & 0.0213 & 0.2214 \\
\hline 3 & 18 & 0.0011 & 0.0133 & 0.2138 \\
\hline 4 & 5 & 0.0008 & 0.0128 & 0.1342 \\
\hline 4 & 14 & 0.0008 & 0.0129 & 0.1382 \\
\hline 5 & 6 & 0.0002 & 0.0026 & 0.0434 \\
\hline 5 & 8 & 0.0008 & 0.0112 & 0.1476 \\
\hline 6 & 7 & 0.0006 & 0.0092 & 0.1130 \\
\hline 6 & 11 & 0.0007 & 0.0082 & 0.1389 \\
\hline 7 & 8 & 0.0004 & 0.0046 & 0.0780 \\
\hline 8 & 9 & 0.0023 & 0.0363 & 0.3804 \\
\hline 9 & 39 & 0.0010 & 0.0250 & 1.2000 \\
\hline 10 & 11 & 0.0004 & 0.0043 & 0.0729 \\
\hline 10 & 13 & 0.0004 & 0.0043 & 0.0729 \\
\hline 13 & 14 & 0.0009 & 0.0101 & 0.1723 \\
\hline 14 & 15 & 0.0018 & 0.0217 & 0.3660 \\
\hline 15 & 16 & 0.0009 & 0.0094 & 0.1710 \\
\hline 16 & 17 & 0.0007 & 0.0089 & 0.1342 \\
\hline 16 & 19 & 0.0016 & 0.0195 & 0.3040 \\
\hline 16 & 21 & 0.0008 & 0.0135 & 0.2548 \\
\hline 16 & 24 & 0.0003 & 0.0059 & 0.0680 \\
\hline 17 & 18 & 0.0007 & 0.0082 & 0.1319 \\
\hline 17 & 27 & 0.0013 & 0.0173 & 0.3216 \\
\hline 21 & 22 & 0.0008 & 0.0140 & 0.2565 \\
\hline 22 & 23 & 0.0006 & 0.0096 & 0.1846 \\
\hline 23 & 24 & 0.0022 & 0.0350 & 0.3610 \\
\hline 25 & 26 & 0.0032 & 0.0323 & 0.5130 \\
\hline 26 & 27 & 0.0014 & 0.0147 & 0.2396 \\
\hline 26 & 28 & 0.0043 & 0.0474 & 0.7802 \\
\hline 26 & 29 & 0.0057 & 0.0625 & 1.0290 \\
\hline 28 & 29 & 0.0014 & 0.0151 & 0.2490 \\
\hline 12 & 11 & 0.0016 & 0.0435 & 0.0000 \\
\hline 12 & 13 & 0.0016 & 0.0435 & 0.0000 \\
\hline 6 & 31 & 0.0000 & 0.0250 & 0.0000 \\
\hline 10 & 32 & 0.0000 & 0.0200 & 0.0000 \\
\hline 19 & 33 & 0.0007 & 0.0142 & 0.0000 \\
\hline 20 & 34 & 0.0009 & 0.0180 & 0.0000 \\
\hline 22 & 35 & 0.0000 & 0.0143 & 0.0000 \\
\hline 23 & 36 & 0.0005 & 0.0272 & 0.0000 \\
\hline 25 & 37 & 0.0006 & 0.0232 & 0.0000 \\
\hline 2 & 30 & 0.0000 & 0.0181 & 0.0000 \\
\hline 29 & 38 & 0.0008 & 0.0156 & 0.0000 \\
\hline 19 & 20 & 0.0007 & 0.0138 & 0.0000 \\
\hline
\end{tabular}

We neglect the reactances of all generators and the transformer tapping when we calculate the coupling strengths, although they are provided in Ref. 41 , because this omission does not affect the results of our numerical experiments, but greatly simplifies the experiments. Thus, the coupling strengths can be calculated by the following steps: (i) using the data in Table IV to calculate the admittance matrix $\mathbf{Y}$, where $Y_{p q}=Y_{q p}=-\frac{1}{R_{p q}+i X_{q p}}$ if $p \neq q$, and $Y_{p p}=-\sum_{q=1}^{N^{0}} Y_{p q}+\frac{1}{2} i B_{p q}$; (ii) using $U$ in Table V to calculate $b_{p q}$ by $b_{p q}=\left|U_{p}\right|\left|U_{q}\right| \Im Y_{p q}$ where $\Im Y_{p q}$ is the imaginary part of $Y_{p q}$.
TABLE V. Bus data. Data from Ref. 41.

\begin{tabular}{|c|c|c|c|c|c|c|c|c|}
\hline Bus No. & $U$ & $D$ & $P_{L}^{0}$ & $P_{L}$ & $P_{G}^{0}$ & $P_{G}$ & Gen. No. & $H$ \\
\hline 1 & 1.0474 & 3 & 0.00 & 0.00 & 0.00 & 0.00 & $\ldots$ & $\ldots$ \\
\hline 2 & 1.0487 & 3 & 0.00 & 0.00 & 0.00 & 0.00 & $\ldots$ & $\ldots$ \\
\hline 3 & 1.0302 & 3 & -322.00 & -323.62 & 0.00 & 0.00 & $\ldots$ & $\ldots$ \\
\hline 4 & 1.0039 & 3 & -500.00 & -501.62 & 0.00 & 0.00 & $\ldots$ & $\ldots$ \\
\hline 5 & 1.0053 & 3 & 0.00 & 0.00 & 0.00 & 0.00 & $\ldots$ & $\ldots$ \\
\hline 6 & 1.0077 & 3 & 0.00 & 0.00 & 0.00 & 0.00 & $\ldots$ & $\ldots$ \\
\hline 7 & 0.9970 & 3 & -233.80 & -235.42 & 0.00 & 0.00 & $\ldots$ & $\ldots$ \\
\hline 8 & 0.9960 & 3 & -522.00 & -523.62 & 0.00 & 0.00 & $\ldots$ & $\ldots$ \\
\hline 9 & 1.0282 & 3 & 0.00 & 0.00 & 0.00 & 0.00 & $\ldots$ & $\ldots$ \\
\hline 10 & 1.0172 & 3 & 0.00 & 0.00 & 0.00 & 0.00 & $\ldots$ & $\ldots$ \\
\hline 11 & 1.0127 & 3 & 0.00 & 0.00 & 0.00 & 0.00 & $\ldots$ & $\ldots$ \\
\hline 12 & 1.0002 & 3 & -7.50 & -9.12 & 0.00 & 0.00 & $\ldots$ & $\ldots$ \\
\hline 13 & 1.0143 & 3 & 0.00 & 0.00 & 0.00 & 0.00 & $\ldots$ & $\ldots$ \\
\hline 14 & 1.0117 & 3 & 0.00 & 0.00 & 0.00 & 0.00 & $\ldots$ & $\ldots$ \\
\hline 15 & 1.0154 & 3 & -320.00 & -321.62 & 0.00 & 0.00 & $\cdots$ & $\ldots$ \\
\hline 16 & 1.0318 & 3 & -329.00 & -330.62 & 0.00 & 0.00 & $\ldots$ & $\ldots$ \\
\hline 17 & 1.0336 & 3 & 0.00 & 0.00 & 0.00 & 0.00 & $\ldots$ & $\ldots$ \\
\hline 18 & 1.0309 & 3 & -158.00 & -159.62 & 0.00 & 0.00 & $\ldots$ & $\ldots$ \\
\hline 19 & 1.0499 & 3 & 0.00 & 0.00 & 0.00 & 0.00 & $\ldots$ & $\ldots$ \\
\hline 20 & 0.9912 & 3 & -628.00 & -629.62 & 0.00 & 0.00 & $\ldots$ & $\ldots$ \\
\hline 21 & 1.0318 & 3 & -274.00 & -275.62 & 0.00 & 0.00 & $\ldots$ & $\ldots$ \\
\hline 22 & 1.0498 & 3 & 0.00 & 0.00 & 0.00 & 0.00 & $\ldots$ & $\ldots$ \\
\hline 23 & 1.0448 & 3 & -247.50 & -249.12 & 0.00 & 0.00 & $\ldots$ & $\ldots$ \\
\hline 24 & 1.0373 & 3 & -308.60 & -310.22 & 0.00 & 0.00 & $\ldots$ & $\ldots$ \\
\hline 25 & 1.0576 & 3 & -224.00 & -225.62 & 0.00 & 0.00 & $\ldots$ & $\ldots$ \\
\hline 26 & 1.0521 & 3 & -139.00 & -140.62 & 0.00 & 0.00 & $\ldots$ & $\ldots$ \\
\hline 27 & 1.0377 & 3 & -281.00 & -282.62 & 0.00 & 0.00 & $\ldots$ & $\ldots$ \\
\hline 28 & 1.0501 & 3 & -206.00 & -207.62 & 0.00 & 0.00 & $\ldots$ & $\ldots$ \\
\hline 29 & 1.0499 & 3 & -283.50 & -285.12 & 0.00 & 0.00 & $\ldots$ & $\ldots$ \\
\hline 30 & 1.0475 & 2 & 0.00 & 0.00 & 250.00 & 248.38 & 10 & 500 \\
\hline 31 & 0.9820 & 2 & -9.20 & -10.82 & 520.81 & 519.19 & 2 & 30.3 \\
\hline 32 & 0.9831 & 2 & 0.00 & 0.00 & 650.00 & 648.38 & 3 & 35.8 \\
\hline 33 & 0.9972 & 2 & 0.00 & 0.00 & 632.00 & 630.38 & 4 & 28.6 \\
\hline 34 & 1.0123 & 2 & 0.00 & 0.00 & 508.00 & 506.38 & 5 & 26.0 \\
\hline 35 & 1.0493 & 2 & 0.00 & 0.00 & 650.00 & 648.38 & 6 & 34.8 \\
\hline 36 & 1.0635 & 2 & 0.00 & 0.00 & 560.00 & 558.38 & 7 & 26.4 \\
\hline 37 & 1.0278 & 2 & 0.00 & 0.00 & 540.00 & 538.38 & 8 & 24.3 \\
\hline 38 & 1.0265 & 2 & 0.00 & 0.00 & 830.00 & 828.38 & 9 & 34.5 \\
\hline 39 & 1.0300 & 2 & -1104.00 & -1105.62 & 1000.00 & 998.38 & 1 & 42.0 \\
\hline
\end{tabular}

${ }^{1}$ P. Pourbeik, P. S. Kundur, and C. W. Taylor, "The anatomy of a power grid blackout," IEEE Power Energy Mag. 4, 22-29 (2006).

${ }^{2}$ D. P. Nedic, I. Dobson, D. S. Kirschen, B. A. Carreras, and V. E. Lynch, "Criticality in a cascading failure blackout model," Int. J. Electron. Power Energy Syst. 28, 627-633 (2006).

${ }^{3}$ G. Andersson et al., "Causes of the 2003 major grid blackouts in North America and Europe, and recommended means to improve system dynamic performance," IEEE Trans. Power Syst. 20, 1922-1928 (2005).

${ }^{4}$ I. Dobson, B. A. Carreras, V. E. Lynch, and D. E. Newman, "Complex systems analysis of series of blackouts: Cascading failure, critical points, and self-organization," Chaos 17, 026103 (2007).

${ }^{5}$ R. Bacher, U. Näf, M. Renggli, W. Bühlmann, and H. Glavitsch, "Report on the blackout in Italy on 28 September 2003," Technical Report, Swiss Federal Office of Energy, 2003.

${ }^{6} \mathrm{C}$. W. Johnson, "Analysing the causes of the Italian and Swiss blackout, 28 th september 2003," in Proceedings of the Twelfth Australian Workshop on Safety Critical Systems and Software and Safety-Related Programmable Systems (Australian Computer Society, 2007), p. 21.

${ }^{7}$ L. L. Lai, H. T. Zhang, S. Mishra, D. Ramasubramanian, C. S. Lai, and F. Y. Xu, "Lessons learned from July 2012 Indian blackout," in Proceedings of the 9th IET International Conference on Advances in Power System Control, Operation and Management (IET, 2012), p. 1. 
${ }^{8}$ L. L. Lai, H. T. Zhang, C. S. Lai, F. Y. Xu, and S. Mishra, "Investigation on July 2012 Indian blackout," in Proceedings of the 2013 International Conference on Machine Learning and Cybernetics (IEEE, 2013), Vol. 1, p. 92.

${ }^{9}$ M. Golshani, G. A. Taylor, I. Pisica, and P. Ashton, "Implementation of wide area monitoring systems and laboratory-based deployment of PMUs," in 2013 48th International Universities' Proceedings of the Power Engineering Conference (UPEC) (IEEE, 2013), pp. 1-6.

${ }^{10}$ Z. Shaobo and S. Zhanhui, "Challenges and opportunities in emergency management of electric power system blackout," in Proceedings of the 2010 International Conference on E-Product E-Service and EEntertainment (IEEE, 2010), p. 1.

${ }^{11}$ J. Shortle, S. Rebennack, and F. W. Glover, "Transmission-capacity expansion for minimizing blackout probabilities," IEEE Trans. Power Syst. 29, 43-52 (2014).

${ }^{12}$ J.-W. Wang and L.-L. Rong, "Cascade-based attack vulnerability on the US power grid," Saf. Sci. 47, 1332-1336 (2009).

${ }^{13}$ P. Henneaux, P.-E. Labeau, and J.-C. Maun, "Blackout probabilistic risk assessment and thermal effects: Impacts of changes in generation," IEEE Trans. Power Syst. 28, 4722-4731 (2013).

${ }^{14}$ J. Giraldo, E. Mojica-Nava, and N. Quijano, "Synchronization of dynamical networks with a communication infrastructure: A smart grid application," in Proceedings of the 2013 IEEE 52nd Annual Conference on Decision and Control (IEEE, 2013), p. 4638.

${ }^{15}$ P. J. Menck, J. Heitzig, J. Kurths, and H. J. Schellnhuber, "How dead ends undermine power grid stability,” Nat. Commun. 5, 3969 (2014).

${ }^{16}$ I. Simonsen, L. Buzna, K. Peters, S. Bornholdt, and D. Helbing, "Transient dynamics increasing network vulnerability to cascading failures," Phys. Rev. Lett. 100, 218701 (2008).

${ }^{17}$ R. V. Solé, M. Rosas-Casals, B. Corominas-Murtra, and S. Valverde, "Robustness of the European power grids under intentional attack," Phys. Rev. E 77, 026102 (2008).

${ }^{18}$ R. Albert, I. Albert, and G. L. Nakarado, "Structural vulnerability of the North American power grid,” Phys. Rev. E 69, 025103 (2004).

${ }^{19}$ P. H. Nardelli, N. Rubido, C. Wang, M. S. Baptista, C. Pomalaza-Raez, P. Cardieri, and M. Latva-aho, "Models for the modern power grid," Eur. Phys. J. Spec. Top. 223, 2423-2437 (2014).

${ }^{20}$ N. Rubido, C. Grebogi, and M. S. Baptista, "Resiliently evolving supplydemand networks," Phys. Rev. E 89, 012801 (2014).

${ }^{21}$ A. Wang, Y. Tang, H. Sun, W. Wu, and J. Yi, “An adaptive emergency control method for interconnected power grids against frequency decline and system blackout," in Proceedings of the 2012 Conference on Power and Energy (IEEE, 2012), p. 439.

${ }^{22}$ B. Enacheanu, M. Fontela, C. Andrieu, H. Pham, A. Martin, and Y. B. Gie-Idea, "New control strategies to prevent blackouts: Intentional islanding operation in distribution networks," in Proceedings of 18th International Conference and Exhibition on Electricity Distribution (IET, 2005), p. 1.

${ }^{23}$ H.-P. Ren, J. Song, R. Yang, M. S. Baptista, and C. Grebogi, "Cascade failure analysis of power grid using new load distribution law and node removal rule," Physica A 442, 239 (2016).
${ }^{24}$ P. Bak, C. Tang, and K. Wiesenfeld, "Self-organized criticality: An explanation of the 1/f noise," Phys. Rev. Lett. 59, 381 (1987).

${ }^{25}$ P. Bak, C. Tang, and K. Wiesenfeld, "Self-organized criticality," Phys. Rev. A 38, 364 (1988).

${ }^{26}$ B. A. Carreras, D. E. Newman, I. Dobson, and A. Poole, "Initial evidence for self-organized criticality in electric power system blackouts," in Proceedings of the 33rd Annual Hawaii International Conference on System Sciences (IEEE, 2000), p. 6.

${ }^{27} \mathrm{M}$. Amin and J. Stringer, "The electric power grid: Today and tomorrow," MRS Bull. 33, 399-407 (2008).

${ }^{28}$ V. Chuvychin, N. Gurov, A. Skutelis, and V. Strelkovs, "Dynamic's problems of frequency and active power control in electric power system," in Proceedings of the 2003 IEEE Bologna Power Tech Conference (IEEE, 2003), Vol. 4, p. 6.

${ }^{29} \mathrm{C}$. Li, Y. Liu, and $\mathrm{H}$. Zhang, "Fast analysis of active powerfrequency dynamics considering network influence," in Proceedings of the 2012 IEEE Power and Energy Society General Meeting (IEEE, 2012), p. 1.

${ }^{30}$ G. Filatrella, A. H. Nielsen, and N. F. Pedersen, "Analysis of a power grid using a Kuramoto-like model,” Eur. Phys. J. B 61, 485-491 (2008).

${ }^{31}$ A. E. Motter, S. A. Myers, M. Anghel, and T. Nishikawa, "Spontaneous synchrony in power-grid networks," Nat. Phys. 9, 191-197 (2013).

${ }^{32}$ S. Y. Caliskan and P. Tabuada, "Towards Kron reduction of generalized electrical networks," Automatica 50, 2586-2590 (2014).

${ }^{33}$ S. Y. Caliskan and P. Tabuada, "Kron reduction of power networks with lossy and dynamic transmission lines," in Proceedings of the 2012 IEEE 51st Annual Conference on Decision and Control (IEEE, 2012), p. 5554.

${ }^{34}$ F. Dörfler and F. Bullo, "Kron reduction of graphs with applications to electrical networks," IEEE Trans. Circuits Syst. I Regul. Pap. 60, 150-163 (2013).

${ }^{35} \mathrm{~F}$. Dörfler, "Dynamics and control in power grids and complex oscillator networks," Ph.D. thesis (University of California Santa Barbara, 2013).

${ }^{36}$ T. Nishikawa and A. E. Motter, "Comparative analysis of existing models for power-grid synchronization,” New J. Phys. 17, 015012 (2015).

${ }^{37}$ H.-I. Su and A. El Gamal, "Modeling and analysis of the role of fastresponse energy storage in the smart grid," in Proceedings of the 49th Annual Allerton Conference on Communication, Control, and Computing (IEEE, 2011), p. 719.

${ }^{38}$ P. F. Ribeiro, B. K. Johnson, M. L. Crow, A. Arsoy, and Y. Liu, "Energy storage systems for advanced power applications," in Proceedings of the IEEE (2001), Vol. 89, p. 1744.

${ }^{39}$ J. D. Glover, M. Sarma, and T. Overbye, Power System Analysis and Design, SI Version (Cengage Learning, 2011).

${ }^{40}$ F. Kühnlenz and P. H. Nardelli, "Dynamics of complex systems built as coupled physical, communication and decision layers," PLoS One 11, e0145135 (2016).

${ }^{41}$ I. Hiskens, "IEEE PES task force on benchmark systems for stability controls," Technical Report, 2013. 